\title{
Kann die Natur helfen, wenn die Ohren klingeln?
}

\author{
Chronischer Tinnitus kann die Lebensqualität erheblich beeinträchtigen; die \\ Behandlung gestaltet sich oft schwierig. Können naturheilkundliche Maß- \\ nahmen bei dieser Störung helfen? Unser Experte Prof. Dr. A.-M. Beer hat \\ einige Tipps parat. Wenn auch Sie Fragen zur Naturheilkunde haben, schrei- \\ ben Sie uns an brigitte.moreano@springer.com.
}

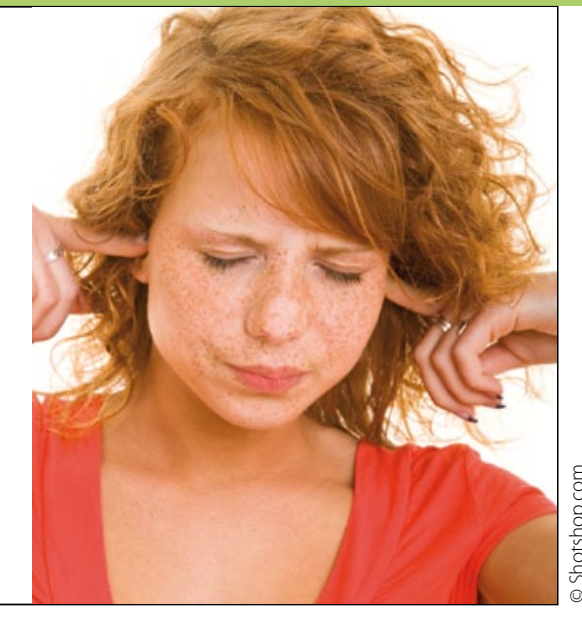

Frage:

Welche Phytopharmaka können bei Tinnitus verordnet werden?

\section{Antwort:}

Unter den verschiedenen Formen des Tinnitus stellt vor allem der chronisch therapieresistente Tinnitus eine Indikation für den Einsatz naturheilkundlicher Maßnahmen dar.

Phytopharmaka sind für diese Indikation am Markt. Ginkgo-biloba-Präparate können erfolgreich eingesetzt werden. Ginkgoblätter verbessern u.a. nachweislich die Mikrozirkulation, hemmen die Thrombozytenaggregation und Erythrozytenadhäsion, steigern die Erythrozytenflexibilität und senken die Blutviskosität. Sie werden zur symptomatischen Behandlung des Tinnitus in einer Dosierung von zweimal bis zu 120 $\mathrm{mg} /$ Tag mindestens acht Wochen lang empfohlen. Die Präparate liegen als Trockenextrakte (35-67:1), Auszugsmittel: Aceton $60 \% \mathrm{~m} / \mathrm{m}$, quantifiziert auf Flavonglykoside, Terpenlactone (Ginkgolide A, B, C, Bilobalid), < 5 ppm Ginkgolsäure vor.

Leichte Magen-Darm-Beschwerden, Kopfschmerzen, Schwindel, allergische Hautreaktionen (Rötung, Schwellung, Juckreiz) können als Nebenwirkungen auftreten. Bei gleichzeitiger Einnahme von gerinnungshemmenden Substanzen, z. B. Antikoagulanzien, ASS oder Clopidogrel, zeigen neue Studien für arzneibuchkonforme Ginkgo-Extrakte keine Interaktionen.

\section{Frage:}

Welche weiteren Maßnahmen bieten sich zur Tinnitusbehandlung an?

\section{Antwort:}

Erfahrungsgemäß bringt eine Blutegelbehandlung - je zwei Blutegel beidseits über den Mastoidknochen - eine deutliche Besserung. Durch die damit verbundene Zuführung u. a. von Hirudin, kann die lokale Durchblutung bis ins Innenohr verbessert werden.

Oftmals sind dauerhafte psychischphysische Belastungen eine wesentliche Mitursache des chronisch rezidivierenden Tinnitus. In der konventionellen Medizin werden psychotherapeutische Behandlungen empfohlen.

Die Ordnungstherapie ist neben der Hydrotherapie, Ernährungstherapie, Phytotherapie und Bewegungstherapie eine der wesentlichen Säulen der klassischen Naturheilverfahren, indem sie die persönliche Lebensordnung des $\mathrm{Pa}$ tienten als gesundheitsrelevanten Faktor betont.

Oft kann durch einfache ordnungstherapeutische Analysen und Beratungen zur Lebensweise der Patienten seine Besserung erreicht werden.

Bei Polyglobulie kann eine isovolämische Hämodilution in Form eines Aderlasses mit anschließender Infusion von Ringerlösung zur Verbesserung der Perfusion vor allem im Bereich der kleinen Arteriolen und Kapillaren zur Anwendung kommen.

Als hypovolämische Hämodilutionstherapie hat der Aderlass Einlass in die konventionelle Medizin gefunden und gehört heute zur Standardtherapie bei Polyzythämie und Hämochromathose. Naturheilkundlich wird meist eine isovolämische Hämodilutionstherapie durchgeführt, bei der die zuerst abgelassene Blutmenge dann durch eine Infusionslösung (z. B. $\mathrm{NaCl}$ 0,9\%) wieder ausgeglichen wird. Aus naturheilkundlicher Sicht ist ein Aderlass ab einem Hämatokritwert von $>40 \mathrm{mg} / \mathrm{dl}$ und Hämoglobinwert von $>16 \%$ indiziert.

Als blutentziehendes, ausleitendes Verfahren wirkt der Aderlass durchblutungsverbessernd. Da es ab einem Hämatokritwert von $>42 \%$ zum Phänomen der Geldrollenbildung kommt, wirkt der Aderlass diesem entgegen und verbessert die Blutrheologie. Die Versorgung der Endstrecken wird verbessert.

Kontraindikationen sind schwere Herzinsuffizienz, Herzklappeninsuffizienz, schlechter Allgemeinzustand, Kachexie, ausgeprägte Anämie, Hypotonie, Exsikkose, allgemeine Erschöpfung sowie Neigung zur Hypotonie.

Prof. Dr. A.-M. Beer, Hattingen = 